\title{
STABILITY OF SOLUTIONS OF LINEAR DELAY DIFFERENTIAL EQUATIONS
}

\author{
M. R. S. KULENOVIC, G. LADAS AND A. MEIMARIDOU
}

ABSTRACT. Consider the linear differential equation

$$
\dot{x}(t)=\sum_{i=1}^{n} p_{i}(t) x\left(t-\tau_{i}\right)=0, \quad t \geqslant t_{0},
$$

where $p_{i} \in C\left(\left[\mathrm{t}_{0}, \infty\right), \mathbf{R}\right)$ and $\tau_{i} \geqslant 0$ for $i=1,2, \ldots, n$. By investigating the asymptotic behavior first of the nonoscillatory solutions of (1) and then of the oscillatory solutions we are led to new sufficient conditions for the asymptotic stability of the trivial solution of (1).

When the coefficients of (1) are all of the same sign, we obtain a comparison result which shows that the nonoscillatory solutions of (1) dominate the growth of the oscillatory solutions.

1. Introduction. In this paper we obtain new stability results for delay differential equations of the form

$$
\dot{x}(t)+\sum_{i=1}^{n} p_{i}(t) x\left(t-\tau_{i}\right)=0, \quad t \geqslant t_{0},
$$

where $p_{i} \in C\left(\left[t_{0}, \infty\right), \mathbf{R}\right)$ and $\tau_{i} \geqslant 0$ for $i=1, \ldots, n$. Our approach is based on dividing the set of solutions of (1) into oscillatory and nonoscillatory solutions and then examining the asymptotic properties of each class.

For equations with one and two delays the same approach was used by Ladas, Sficas, and Stavroulakis [7] and Ladas and Sficas [5] respectively.

When the coefficients of (1) are all of the same sign, we obtain a comparison result which shows that the nonoscillatory solutions of (1) dominate the growth of the oscillatory solutions.

In the sequel, for convenience, we will assume that inequalities concerning values of functions are satisfied eventually, that is for all large $t$.

2. Asymptotic behavior of oscillatory and nonoscillatory solutions. Without loss of generality, we will assume throughout this paper that

$$
0 \leqslant \tau_{1}<\tau_{2}<\cdots<\tau_{n} .
$$

Our first result gives sufficient conditions for the nonoscillatory solutions of (1) to tend to zero as $t \rightarrow \infty$.

Received by the editors November 12, 1985 .

1980 Mathematics Subject Classification (1985 Revision). Primary 34K25; Secondary 34K15.

Key words and phrases. Linear delay differential equations, stability of solutions, asymptotically stable. 
THEOREM 1. Consider the DDE (1) and assume that (2) holds and that there exist positive constants $A$ and $B$ such that the following conditions are satisfied for sufficiently large $t$.

$$
\begin{gathered}
\left|p_{i}(t)\right| \leqslant A \quad \text { for } i=1,2, \ldots, n, \\
\sum_{i=1}^{n} p_{i}(t) \geqslant B
\end{gathered}
$$

and

$$
\sum_{i=1}^{n-1} \int_{t-\tau_{n}}^{t-\tau_{i}}\left|p_{i}\left(s+\tau_{i}\right)\right| d s \leqslant 1 .
$$

Then every nonoscillatory solution of (1) tends to zero as $t \rightarrow \infty$.

Proof. Let $x(t)$ be a nonoscillatory solution of (1). Since the negative of a solution of $(1)$ is also a solution we will suppose that $x(t)>0$. Set

$$
z(t)=x(t)+\sum_{i=1}^{n-1} \int_{t-\tau_{n}}^{t-\tau_{i}} p_{i}\left(s+\tau_{i}\right) x(s) d s .
$$

with the convention that when $n=1$ the above sum is zero. Then

$$
\begin{aligned}
\dot{z}(t) & =-\left[\sum_{i=1}^{n} p_{i}\left(t-\tau_{n}+\tau_{i}\right)\right] x\left(t-\tau_{n}\right) \\
& \leqslant-B x\left(t-\tau_{n}\right),
\end{aligned}
$$

which implies that $z(t)$ is a strictly decreasing function. Set

$$
L=\lim _{t \rightarrow \infty} z(t) \text {. }
$$

We claim that $L \in \mathbf{R}$. Otherwise $L=-\infty$ and, because of (5), $x(t)$ must be unbounded. Choose a $t_{1} \geqslant t_{0}+\tau_{n}$ in such a way that (5) is satisfied for $t \geqslant t_{1}$, $z\left(t_{1}\right)<0$, and

$$
x\left(t_{1}\right)=\max _{t_{0} \leqslant s \leqslant t_{1}} x(s) .
$$

Clearly, this choice of $t_{1}$ is possible because $x(t)$ is unbounded. Then,

$$
\begin{aligned}
0 & >z\left(t_{1}\right)=x\left(t_{1}\right)+\sum_{i=1}^{n-1} \int_{t_{1}-\tau_{n}}^{t_{1}-\tau_{i}} p_{i}\left(s+\tau_{i}\right) x(s) d s \\
& \geqslant x\left(t_{1}\right)-\sum_{i=1}^{n-1} \int_{t_{1}-\tau_{n}}^{t_{1}-\tau_{i}}\left|p_{i}\left(s+\tau_{i}\right)\right| x(s) d s \\
& \geqslant x\left(t_{1}\right)\left[1-\sum_{i=1}^{n-1} \int_{t_{1}-\tau_{n}}^{t_{1}-\tau_{i}}\left|p_{i}\left(s+\tau_{i}\right)\right| d s\right] \\
& \geqslant 0
\end{aligned}
$$

which is a contradiction. We are now ready to prove that $\lim _{t \rightarrow \infty} x(t)=0$. In fact, integrating (8) from $t_{1}$ to $t$, for $t_{1}$ sufficiently large, and letting $t \rightarrow \infty$, we find

$$
L-z\left(t_{1}\right) \leqslant-B \int_{t_{1}}^{\infty} x\left(s-\tau_{n}\right) d s .
$$


Hence $x \in L^{1}\left[t_{1}, \infty\right)$. From (1) and (3) it then follows that $\dot{x} \in L^{1}\left[t_{1}, \infty\right)$. Therefore, $\lim _{t \rightarrow \infty} x(t)$ exists and it has to be zero because $x \in L^{1}\left[t_{1}, \infty\right)$. The proof is complete.

For conditions concerning the existence of nonoscillatory solutions see [8].

Our next result deals with the asymptotic equivalence to zero of all oscillatory solutions of (1).

THEOREM 2. Consider the DDE (1) and assume that (2) holds and that eventually,

$$
\begin{gathered}
\sum_{i=1}^{n} p_{i}\left(t-\tau_{n}+\tau_{i}\right) \neq 0, \\
\sum_{i=1}^{n-1} \int_{t-\tau_{n}}^{t-\tau_{i}}\left|p_{i}\left(s+\tau_{i}\right)\right| d s \leqslant Q_{1},
\end{gathered}
$$

and

$$
\sum_{i=1}^{n} \int_{t-\tau_{n}}^{t}\left|p_{i}\left(s-\tau_{n}+\tau_{i}\right)\right| d s \leqslant Q_{2},
$$

where $Q_{1}$ and $Q_{2}$ are positive constants such that

$$
2 Q_{1}+Q_{2}<1 \text {. }
$$

Then every oscillatory solution of (1) tends to zero as $t \rightarrow \infty$.

PRoof. Let $x(t)$ be an oscillatory solution of (1). First we will prove that $x(t)$ is bounded. Otherwise, $x(t)$ is unbounded. Choose $t_{1} \geqslant t_{0}+\tau_{n}$ such that (9)-(11) hold for $t \geqslant t_{1}$ and also

$$
\max _{t_{1} \leqslant s \leqslant t}|x(s)| \geqslant \max _{t-\tau_{n} \leqslant s \leqslant t-\tau_{1}}|x(s)|, \quad \text { for } t \geqslant t_{1} .
$$

Clearly, this choice of $t_{1}$ is possible because $x(t)$ is unbounded. Then, from (6), we have

which implies that

$$
\begin{aligned}
|z(t)| & \geqslant|x(t)|-\sum_{i=1}^{n-1} \int_{t-\tau_{n}}^{t-\tau_{i}}\left|p_{i}\left(s+\tau_{i}\right)\right||x(s)| d s \\
& \geqslant|x(t)|-\left[\max _{t_{1} \leqslant s \leqslant t}|x(s)|\right] Q_{1}
\end{aligned}
$$

$$
\max _{t_{1} \leqslant s \leqslant t}|z(s)| \geqslant\left[1-Q_{1}\right] \max _{t_{1} \leqslant s \leqslant t}|x(s)|>0 .
$$

Hence, $z(t)$ is unbounded. Also, from (7) we see that $\dot{z}(t)$ oscillates. Thus, there exists a sequence of points $\left\{\xi_{k}\right\}$ such that $\xi_{k} \geqslant t_{1}$ for $k=1,2, \ldots, \lim _{k \rightarrow \infty} \xi_{k}=\infty$, $\lim _{k \rightarrow \infty}\left|z\left(\xi_{k}\right)\right|=\infty, \dot{z}\left(\xi_{k}\right)=0$ for $k=1,2, \ldots$ and

$$
\left|z\left(\xi_{k}\right)\right|=\max _{t_{1} \leqslant s \leqslant \xi_{k}}|z(s)| .
$$

From (7), using Condition (9) and the fact that $\dot{z}\left(\xi_{k}\right)=0$, we see that $x\left(\xi_{k}-\tau_{n}\right)=0$ for $k=1,2, \ldots$ and so (6) yields

$$
z\left(\xi_{k}-\tau_{n}\right)=\sum_{i=1}^{n-1} \int_{\xi_{k}-2 \tau_{n}}^{\xi_{k}-\tau_{n}-\tau_{i}} p_{i}\left(s+\tau_{i}\right) x(s) d s, \quad k=1,2, \ldots
$$


Integrating (7) from $\xi_{k}-\tau_{n}$ to $\xi_{k}$ and using (14) we obtain

$z\left(\xi_{k}\right)=\sum_{i=1}^{n-1} \int_{\xi_{k}-2 \tau_{n}}^{\xi_{k}-\tau_{n}-\tau_{i}} p_{i}\left(s+\tau_{i}\right) x(s) d s-\int_{\xi_{k}-\tau_{n}}^{\xi_{k}}\left[\sum_{i=1}^{n} p_{i}\left(s-\tau_{n}+\tau_{i}\right)\right] x\left(s-\tau_{n}\right) d s$.

Using (10) and (11) we find from (15) that

$$
\left|z\left(\xi_{k}\right)\right| \leqslant\left(Q_{1}+Q_{2}\right) \max _{t_{1} \leqslant s \leqslant \xi_{n}}|x(s)|
$$

and, in view of (13)

$$
\left(1-Q_{1}\right) \max _{t_{1} \leqslant s \leqslant \xi_{n}}|x(s)| \leqslant\left(Q_{1}+Q_{2}\right) \max _{t_{1} \leqslant s \leqslant \xi_{n}}|x(s)| .
$$

This implies that

$$
1 \leqslant 2 Q_{1}+Q_{2}
$$

which contradicts (12) and proves our claim that every oscillatory solution of (1) is bounded. To complete the proof it remains to show that every bounded and oscillatory solution $x(t)$ of (1) tends to zero as $t \rightarrow \infty$. Otherwise

$$
\mu \equiv \limsup _{t \rightarrow \infty}|x(t)|>0
$$

and for any $\varepsilon>0$ there exists a $t_{2} \geqslant t_{1}$ such that

$$
|x(t)|<\mu+\varepsilon, \quad t \geqslant t_{2} .
$$

From (6) we have

$$
\begin{aligned}
|z(t)| & \geqslant|x(t)|-\sum_{i=1}^{n-1} \int_{t-\tau_{n}}^{t-\tau_{i}}\left|p_{i}\left(s+\tau_{i}\right)\right||x(s)| d s \\
& \geqslant|x(t)|-(\mu+\varepsilon) Q_{1}, \quad t \geqslant t_{2} .
\end{aligned}
$$

Thus

$$
\alpha \equiv \limsup _{t \rightarrow \infty}|x(t)| \geqslant \mu-(\mu+\varepsilon) Q_{1} .
$$

As $\varepsilon$ is arbitrary, it follows that

$$
\alpha \geqslant \mu\left(1-Q_{1}\right)>0 .
$$

Since $\dot{z}(t)$ oscillates, there exists a sequence of points $\left\{\zeta_{k}\right\}$ such that $\zeta_{k} \geqslant t_{2}$ for $n=1,2, \ldots, \lim _{k \rightarrow \infty} \zeta_{k}=\infty, \dot{z}\left(\zeta_{k}\right)=0$ for $k=1,2, \ldots$ and

$$
\lim _{k \rightarrow \infty}\left|z\left(\zeta_{k}\right)\right|=\alpha \text {. }
$$

Also (14) and so (15) is true with $\xi_{k}$ replaced by $\zeta_{k}$. Hence, from (15),

$$
\begin{aligned}
\left|z\left(\zeta_{k}\right)\right| & \leqslant(\mu+\varepsilon)\left[\sum_{i=1}^{n-1} \int_{\zeta_{k}-2 \tau_{n}}^{\zeta_{k}-\tau_{n}-\tau_{i}}\left|p_{i}\left(s+\tau_{i}\right)\right| d s+\sum_{i=1}^{n} \int_{\zeta_{k}-\tau_{n}}^{\zeta_{k}}\left|p_{i}\left(s-\tau_{n}+\tau_{i}\right)\right| d s\right] \\
& \leqslant(\mu+\varepsilon)\left(Q_{1}+Q_{2}\right)
\end{aligned}
$$


and so as $\varepsilon$ is arbitrary

$$
\mu\left(1-Q_{1}\right) \leqslant \alpha \leqslant \mu\left(Q_{1}+Q_{2}\right)
$$

or

$$
1 \leqslant 2 Q_{1}+Q_{2},
$$

which contradicts the hypothesis (12). The proof is complete.

Combining Theorems 1 and 2, we obtain the following result.

THeOREM 3. Consider the DDE (1) and assume that (2)-(4) and (10)-(12) are satisfied. Then the trivial solution of (1) is globally asymptotically stable.

When the coefficients $p_{i}(t)$ of $(1)$ are constants, that is for the DDE

$$
\dot{x}(t)+\sum_{i=1}^{n-1} p_{i} x\left(t-\tau_{i}\right)=0, \quad t \geqslant t_{0},
$$

Theorems 1, 2, and 3 reduce to the following corollaries.

Corollary 1. Consider the DDE (16) and assume that (2) holds,

$$
\sum_{i=1}^{n} p_{i}>0
$$

and

$$
\sum_{i=1}^{n-1}\left(\tau_{n}-\tau_{i}\right)\left|p_{i}\right| \leqslant 1 .
$$

Then every nonoscillatory solution of (16) tends to zero as $t \rightarrow \infty$.

Corollary 2. Consider the DDE (16) and assume that (2) holds,

$$
\sum_{i=1}^{n} p_{i} \neq 0
$$

and

$$
\sum_{i=1}^{n}\left(3 \tau_{n}-2 \tau_{i}\right)\left|p_{i}\right|<1
$$

Then every oscillatory solution of (16) tends to zero as $t \rightarrow \infty$.

Corollary 3. Consider the DDE (16) and assume that (2), (17), and (20) hold. Then the trivial solution of (16) is globally asymptotically stable.

REMARK 1. When the delays of (16) are all zero, that is in the case of the ordinary differential equation

$$
\dot{x}(t)+\left(\sum_{i=1}^{n} p_{i}\right) x(t)=0 .
$$

every nontrivial solution is nonoscillatory. In this case, (17) is the only hypothesis needed for Corollary 1 to hold. Also (17) is exactly what is needed for every solution of (21) to tend to zero as $t \rightarrow \infty$. In this sense our Theorem 1 is a sharp result. 
REMARK 2. Combining Theorem 2 (or Corollary 2) with known oscillation results we may obtain various stability theorems for (1) or (16). For example, using the results of Hunt and Yorke [3] and Ladas and Stavroulakis [6] we get the following.

COROllary 4. Consider the DDE (16) and assume that the coefficients $p_{i}$ are all positive and that conditions (2) and (20) hold. Then, either of the additional conditions

$$
\sum_{i=1}^{n} p_{i} \tau_{i}>\frac{1}{e}
$$

or

$$
\left(\sum_{i=1}^{n} \tau_{i}\right)\left(\prod_{i=1}^{n} p_{i}\right)^{1 / n}>\frac{1}{e}
$$

implies that the trivial solution of (16) is globally asymptotically stable.

Remark 3. Consider the DDE

$$
\dot{x}(t)+a x(t)+b x(t-\tau)=0,
$$

where $a, b \in \mathbf{R}$ and $\tau>0$. A result of Krasovskii [4] (see also Driver [1]) states that

$$
a>0 \text { and }|b|>a
$$

imply that every solution of (22) tends to zero as $t \rightarrow \infty$. On the other hand, our Corollary 3 implies that if

$$
a+b>0 \text { and } \tau(3|a|+|b|)<1,
$$

then every solution of (22) tends to zero as $t \rightarrow \infty$. Clearly, conditions (23) and (24) are not compatible.

3. Comparison results and stability. The following lemma about solutions of (1) will be useful in this section.

LEMma 1. Let $z(t)$ be a nonoscillatory solution of (1). Set

$$
w(t)=x(t) / z(t), \quad t \geqslant T,
$$

where $x(t)$ is any solution of (1) and $T \geqslant t_{0}$ is such that $z(t) \neq 0$, for $t \geqslant T$. Then,

$$
\dot{w}(t)=\sum_{i=1}^{n} p_{i}(t) \frac{z\left(t-\tau_{i}\right)}{z(t)}\left[w(t)-w\left(t-\tau_{i}\right)\right], \quad t \geqslant T .
$$

ProOF.

$$
\begin{aligned}
\dot{w}(t) & =\frac{1}{z^{2}(t)}\left[-z(t) \sum_{i=1}^{n} p_{i}(t) x\left(t-\tau_{i}\right)+x(t) \sum_{i=1}^{n} p_{i}(t) z\left(t-\tau_{i}\right)\right] \\
& =\frac{1}{z^{2}(t)} \sum_{i=1}^{n} p_{i}(t)\left[\frac{x(t)}{z(t)}-\frac{x\left(t-\tau_{i}\right)}{z\left(t-\tau_{i}\right)}\right] z(t) z\left(t-\tau_{i}\right) \\
& =\sum_{i=1}^{n} p_{i}(t) \frac{z\left(t-\tau_{i}\right)}{z(t)}\left[w(t)-w\left(t-\tau_{i}\right)\right] .
\end{aligned}
$$

For equations with one delay the transformation (25) was used by Nosov. See [2]. 
In the next theorem we will assume that the coefficients $p_{i}(t)$ of (1) satisfy one of the following conditions for sufficiently large $t$ : Either

$$
p_{i}(t) \geqslant 0 \text { for } i=1,2, \ldots \text { and } \sum_{i=1}^{n} p_{i}(t)>0,
$$

or

$$
p_{i}(t) \leqslant 0 \text { for } i=1,2, \ldots \text { and } \sum_{i=1}^{n} p_{i}(t)<0 .
$$

THEOREM 4. Assume that either (27) or (28) is satisfied. Let $z(t)$ be a nonoscillatory solution of (1) and let $x(t)$ be any oscillatory solution. Then there exists $k>0$ such that eventually

$$
|x(t)| \leqslant k|z(t)|
$$

Proof. Assume $z(t)>0$ for $t \geqslant T$. The case $z(t)<0$ for $t \geqslant T$ can be treated in a similar way. Using the function $w$ introduced in Lemma 1 we have to prove that $w$ is bounded. Otherwise, since $w$ is an oscillatory function, there exists $t^{*} \geqslant T+\tau_{n}$ such that $\dot{w}\left(t^{*}\right)=0$ and either

$$
w\left(t^{*}\right)>w(s) \text { for } T \leqslant s<t^{*},
$$

or

$$
w\left(t^{*}\right)<w(s) \text { for } T \leqslant s<t^{*} .
$$

Substituting $t=t^{*}$ into (26), we get a contradiction.

The next result compares nonoscillatory solution with oscillatory solutions as well as with other nonoscillatory solutions.

THEOREM 5. Assume that (28) is satisfied. Then for every solution $x(t)$ of $(1)$ there exists $k>0$ such that eventually

$$
|x(t)| \leqslant k|\dot{z}(t)|
$$

Proof. In view of the preceding theorem it suffices to assume that $x(t)$ and $z(t)$ are positive for $t \geqslant T \geqslant t_{0}$. We should prove that $w(t)$ is bounded. Otherwise, there exists $t^{*} \geqslant T+\tau_{n}$ such that

$$
\dot{w}\left(t^{*}\right) \geqslant 0 \text { and } w\left(t^{*}\right)>w(s) \text { for } T \leqslant s<t^{*} .
$$

Setting $t=t^{*}$ in (26) we get a contradiction.

Using Theorem 4 we obtain the following stability result.

THEOREM 6. Assume that (27) is satisfied and that

$$
\int_{t_{0}}^{\infty} \sum_{i=1}^{n} p_{i}(t) d t=\infty
$$

Furthermore, assume that (1) has a nonoscillatory solution. Then the trivial solution of (1) is globally asymptotically stable.

Proof. In view of Theorem 4 it suffices to prove that every nonoscillatory solution of (1) tends to zero as $t \rightarrow \infty$. Otherwise, (1) has a solution $z(t)$ such that

$$
z(t)>0, \quad \dot{z}(t) \leqslant 0 \text {. }
$$


Let

$$
l \equiv \lim _{t \rightarrow \infty} z(t)>0
$$

Then

$$
\begin{aligned}
0 & =\dot{z}(t)+\sum_{i=1}^{n} p_{i}(t) z\left(t-\tau_{i}\right) \\
& \geqslant \dot{z}(t)+\frac{l}{2} \sum_{i=1}^{n} p_{i}(t) .
\end{aligned}
$$

Integrating from $t_{1}$ to $t$, with $t_{1}$ sufficiently large, and letting $t \rightarrow \infty$, we find

$$
l-z\left(t_{1}\right)+\frac{l}{2} \int_{t_{1}}^{\infty} \sum_{i=1}^{n} p_{i}(t) d t \leqslant 0 .
$$

This contradiction completes the proof.

REMARK 4. The hypotheses of Theorem 6 are satisfied, for example, when the coefficients $p_{i}$ of (1) are positive constants and the characteristic equation

$$
\lambda+\sum_{i=1}^{n} p_{i} e^{-\lambda \tau_{i}}=0
$$

has a real root. When the coefficients $p_{i}(t)$ are variables, Ladas, Sficas and Stavroulakis [7] have given sufficient conditions for (1) to have nonoscillatory solutions. Combining this result with Theorem 6, we obtain the following stability result.

Corollary 5. Assume that (27) and (29) are satisfied and that the coefficients of (1) have bounded derivatives. Furthermore, assume that there exist constants $p_{i}$ such that

$$
p_{i}(t) \leqslant p_{i} \quad \text { for } i=1,2, \ldots, n
$$

and that (3) has a real root. Then the trivial solution of (1) is asymptotically stable.

In the case of constant coefficients, Theorems 4 and 5 imply the following results for the corresponding characteristic equation (30).

Corollary 6. Assume that $p_{i}<0$ for $i=1,2, \ldots, n$. Then (30) has exactly one real root $\lambda_{0}$, and for any other root $\lambda$ of (30),

$$
\operatorname{Re} \lambda \leqslant \lambda_{0} \text {. }
$$

COROllary 7. Assume that $p_{i}>0$ for $i=1,2, \ldots, n$. Then either (30) has no real roots or it has a negative real root $\lambda_{0}$ such that $\operatorname{Re} \lambda \leqslant \lambda_{0}$, for any nonreal root $\lambda$ of (30).

\section{REFERENCES}

1. R. D. Driver, Exponential decay in some linear delay differential equations, Amer. Math. Monthly 85 (1978), 757-760.

2. L. E. El'sgol'c and S. B. Norkin, Introduction to the theory of differential equations with deviating argument, Holden-Day, San Francisco, Calif., 1966 (translated from Russian).

3. B. R. Hunt and J. A. Yorke, When all solutions of $x^{\prime}=-\sum_{i=1}^{n} q_{i}(t) x\left(t-T_{i}(t)\right)$ oscillate, J. Differential Equations 53 (1984), 139-145. 
4. N. N. Krasovskiī, Stability of motion, Stanford Univ. Press, Stanford, Calif., 1963 (translated from Russian).

5. G. Ladas and Y. G. Sficas, Asymptotic behavior of oscillatory solutions, Proc. Internat. Conf. on Theory and Applications of Differential Equations (Pan American University, Edinburg, Texas, May 20-23, 1985).

6. G. Ladas and I. P. Stavroulakis, Oscillations caused by several retarded and advanced arguments, J. Differential Equations 44 (1982), 134-152.

7. G. Ladas, Y. G. Sficas and I. P. Stavroulakis, Asymptotic behavior of solutions of retarded differential equations, Proc. Amer. Math. Soc. 88 (1983), 247-253.

8. G. Ladas, Y. G. Sficas and I. P. Stavroulakis, Nonoscillatory functional differential equations, Pacific J. Math. 115 (1984), 391-398.

Department of Mathematics, University of Sarajevo, Sarajevo 71000, Yugoslavia

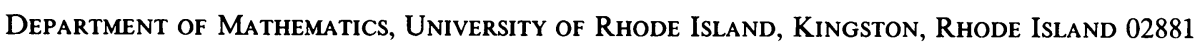

Department of Electrical Engineering, Democritus University of Thrace, Xanthi 67100 , GREECE 